\title{
New enhancements and validation of force based posture and discomfort predictions
}

\author{
Hans-Joachim Wirsching ${ }^{\mathrm{a},{ }^{*}}$ and Florian Engstler ${ }^{\mathrm{b}}$ \\ ${ }^{a}$ Ergonomic Simulation Department, Human Solutions, Europaallee 10, 67657 Kaiserslautern, Germany \\ ${ }^{\mathrm{b}}$ Chair of Ergonomics, Technical University Munich, Boltzmannstraße 15, 85747 Garching, Germany
}

\begin{abstract}
Many digital human models provide the crucial method to calculate postures in virtual environments. Traditionally these methods refer to experiments and make use of statistical modeling. They provide sufficient results in their specialized domain, but cannot be used in general applications. In addition they do not consider important design aspects as forces and discomfort. Hence a new force based approach has been introduced in order to overcome these shortcomings. Based on biomechanical models of active maximal and passive receding joint torques, a mechanical optimization generates static stable postures and related discomfort ratings. The results were promising, but showed some model deficiencies and were not validated in detail. The present paper continues this work and provides necessary model enhancements. Finally the new prediction models are validated on real experiments. The validation results are presented and discussed regarding to usability aspects and future development work.
\end{abstract}

Keywords: biomechanics, digital human model, generic simulation model, validation

\section{Introduction}

One of the most important features of digital human models is the automatic posture prediction in virtual environments. These methods help designers to efficiently get reliable task specific postures for human design analyses.

Classical methods are based on experiments, statistical models and inverse kinematics $[1,4]$. They proof sufficient validity for tasks similar to the experiments, but show inaccuracies for other tasks [3]. Additionally they do not consider forces and stability aspects, which are important in design evaluations. Moreover, they do not provide discomfort assessments without extending the experimental efforts [5].

A new approach for static posture and discomfort prediction has been proposed and discussed to overcome these shortcomings [6]. This general approach is based on measured human maximal and receding joint torque data and an optimization method to minimize joint loads and to provide stable postures and discomfort ratings.
This approach has been implemented in RAMSIS and evaluated on several tasks (driving, hand brake operation, box lifting, drilling). The evaluation showed visual reasonable posture and discomfort results, but could not proof validity due to the lack of missing real posture and discomfort data. In addition principle deficiencies in the force coupling of the manikin with the environment and in the consideration of passive receding torques in the posture prediction models were identified.

The present paper describes the overall simulation approach and methods in Section 2. In Section 2.1 the main model components are recapitulated and the new enhancements are given in Section 2.2. The updated model has been integrated in RAMSIS and validated by experiments presented in Section 3. The validation results are presented in Section 4 and finally discussed in Section 5 .

\footnotetext{
${ }^{*}$ Hans-Joachim Wirsching. E-mail: hans-joachim.wirsching@human-solutions.com.
} 


\section{Simulation approach and methods}

\subsection{Main model components}

The model approach is exhaustively described in [6]. It is based on the assumption that humans try to perform tasks with less effort, less strain and less discomfort by considering physical restrictions like stability and feasibility. In particular a human optimizes unconsciously the posture to use as less muscle forces as possible.

In a human body muscles generate forces and produce active joint torques to balance the posture between internal body weights and external affecting forces. These torques are restricted to maximal joint torques feasible along the joint variation ranges by the human. The joint load is defined as ratio between active and maximal torque (along active torque direction) and provides an indicator for discomfort ratings. In addition the joints are subjected to passive (receding) torques resulting from internal tensions of soft tissue, ligaments and tendons.

All these aspects are integrated in a biomechanical model illustrated in Figure 1. They are supplemented by the limb mass and center of gravity prediction in RAMSIS.

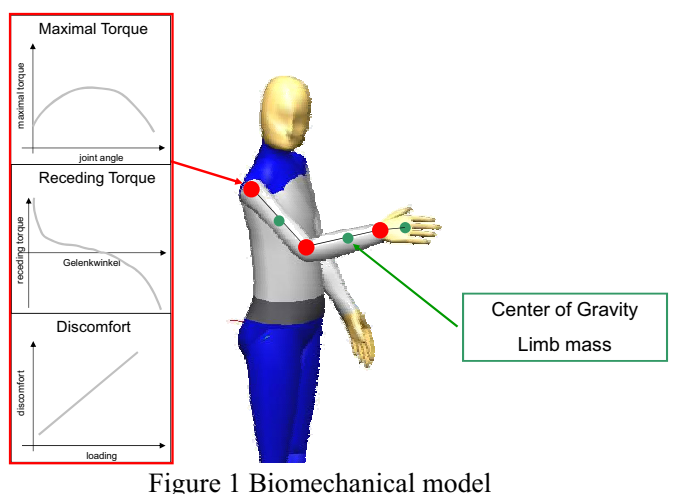

A critical part is the model of the maximal and receding torques. The torque amplitudes are calculated by functions for each joint and for each degree of freedom of the joint. This leads to interpolated 3D ellipsoids representing the maximal torques in any direction and to the zero-g posture for zero passive receding torques (Figure 2 ).
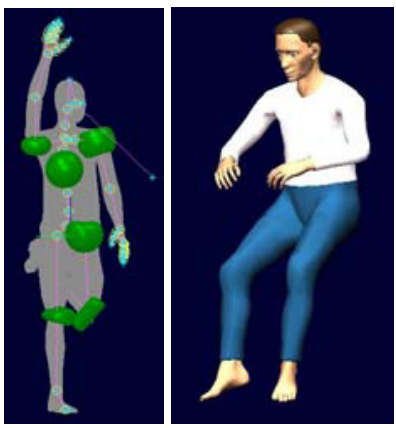

Figure 2 Maximal torque ellipsoids and zero-g posture

These posture dependent joint torque amplitude functions are based on measurements on real subjects (Figure 3). The maximum torques were measured in each joint and each direction by enforcing the subject to apply maximum forces. The receding torques were measured while supporting the limbs to compensate the gravity. The results were structured in a gender and age group specific database.
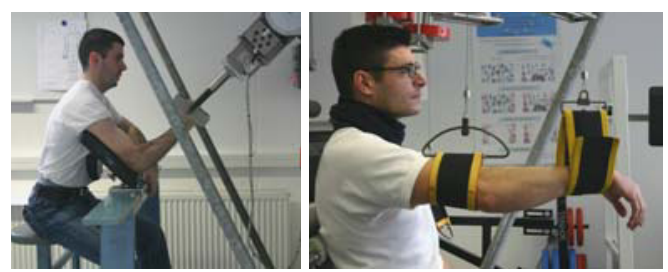

Figure 3 Joint torque measurements (maximal left, receding right)

The biomechanical model is integrated in a mechanical model coupling the manikin with the environment taking into account body weights $\mathrm{G}$, external forces $E$ and support forces $F$ (Figure 4).

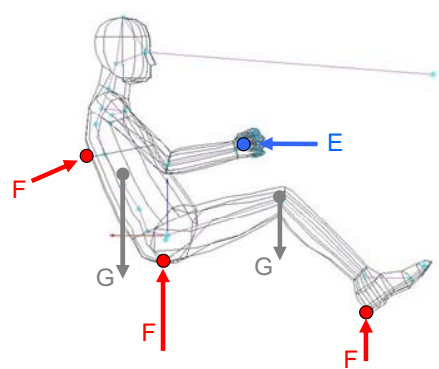

Figure 4 Mechanical model

This model guarantees the static equilibrium with respect to external and internal forces.

Finally the biomechanical and mechanical models are combined with an inverse kinematic method to predict postures and support forces with respect to user defined geometrical constraints by minimizing the joint loads according to the objective function in Equation 1. 
Equation 1 Objective function

$$
\min \phi:=\sum_{i}\left(\frac{\left\|M_{\text {Active }}\right\|}{M_{\text {Maximal }}}\right)^{2}+\left(\frac{\left\|M_{\text {Passive }}\right\|}{M_{\text {Maximal }}}\right)^{2}
$$

The joint loads in the final posture are directly extracted from the posture optimization results (Equation 2) and range in the interval $[0,1]$. Equation 2 Joint load definition

$$
L:=\max \left(\frac{\left\|M_{\text {Active }}\right\|}{M_{\text {Maximal }}}, \frac{\left\|M_{\text {Passive }}\right\|}{M_{\text {Maximal }}}\right)
$$

The discomfort rating of a body part is calculated as maximal joint load of the corresponding joints. Analogue the total body rating is taken as maximal rating of all body parts.

In summary the discomfort model provides ratings in the interval $[0,1]$ and is compatible to the posture prediction model.

\subsection{Model enhancements}

The original model (Section 2.1) is improved by the following aspects.

\subsubsection{Passive torque model}

The original influence of the active joint loads dominated the passive forces (Equation 1) due to larger amplitudes, which often leads to unreasonable postures at the limits of the anatomical joint ranges.

This drawback has been removed by two related actions. First the modeling on the original experimental data [2] has been stabilized. Instead of a cubic regression a strongly monotone and normalized cubic polynomial is fitted into the data. The monotone function gives \pm 1 at the joint range boundaries and 0 at the zero-g position of the joint (Figure 5).

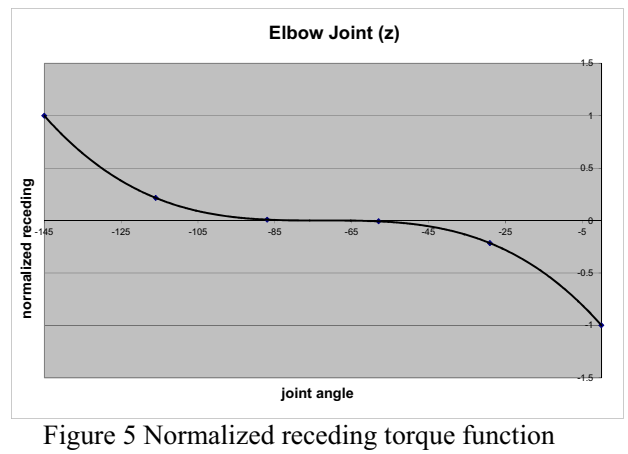

In a second step the maximal torque denominator for normalized passive receding torques in Equation 1 has been replaced by a constant factor $\mathrm{c}$, such that the prediction is reasonable for a wide range of applications (Equation 3).

Equation 3 Adjusted object function

$$
\min \phi:=\sum_{i}\left(\frac{\left\|M_{\text {Active }}\right\|}{M_{\text {Maximal }}}\right)^{2}+c\left\|M_{\text {Passive }}\right\|^{2}
$$

\subsubsection{Force coupling restrictions}

The original prediction of support forces is just based on minimizing joint loads, without considering characteristics of the environment to transfer support forces (Figure 4). This led in some applications to unreasonable force directions.

This shortcoming has been overcome by specific user defined coupling restrictions for environmental conditions like normal and tangential directions along surfaces, which are considered by the prediction method (Figure 6). This helps to define problems with more realistic boundary conditions.
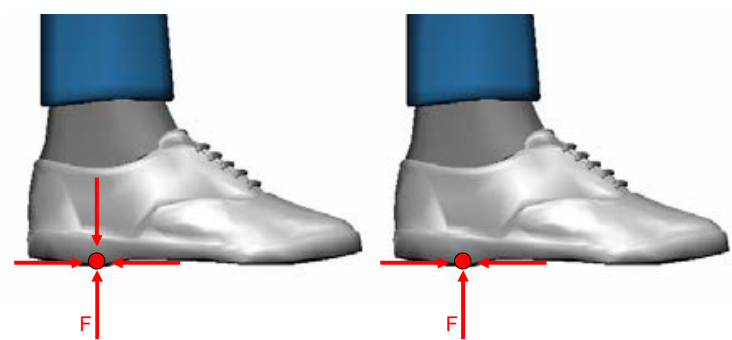

Figure 6 Arbitrary (left) and environment specific (right) support force directions

\section{Validation experiments}

In order to poof the validity of the methods two automotive application scenarios have been defined (Figure 7). The first case addresses the task of closing a car door in two different opening angles while seating (case "door"). The second case covers the task of lifting a box with two different weights (case "box"). 


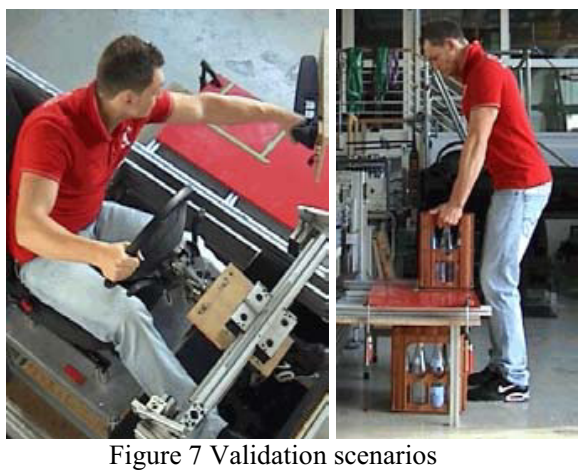

The validation did not address the entire dynamic motion, but focused on the most critical static posture at the beginning of each motion, i.e. start moving the door / lifting the box).

Both scenarios define four tasks in total, which were performed by 20 test subjects. For each trial the posture was measured by calibrated photographs and discomfort ratings were collected on CP50 scale when the subjects apply force to lift the box or close the door (static capture). The test subjects were divided into four groups of young and old large males and small females respectively (German population) to cover different anthropometrics and strengths (Figure 8).

\begin{tabular}{|c|c|c|}
\hline & 20-30 years & $50-70$ years \\
\hline $\mathrm{M}_{95}$ & $\begin{array}{l}\text { PG1 } \\
5 \text { VPs }\end{array}$ & $\begin{array}{c}\text { PG2 } \\
5 \text { VPs }\end{array}$ \\
\hline $\mathrm{F}_{05}$ & $\begin{array}{c}\text { PG3 } \\
5 \text { VPs }\end{array}$ & $\begin{array}{c}\text { PG4 } \\
5 \text { VPs }\end{array}$ \\
\hline
\end{tabular}

The environmental and anthropometrical conditions of the experiments are modeled in a virtual environment and the corresponding posture and discomfort ratings are calculated for each trial (Figure 9).

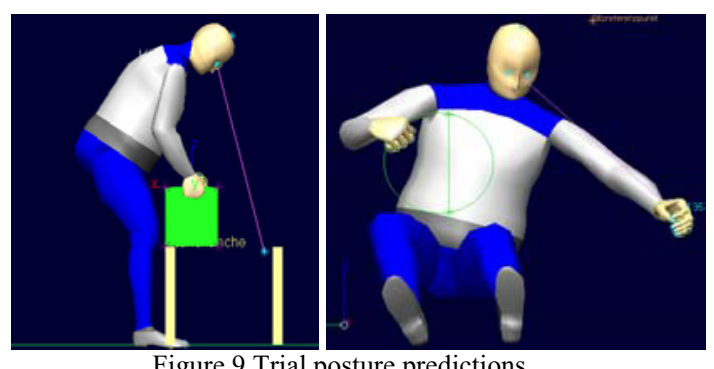

Figure 9 Trial posture predictions
These results are validated on the measurements of the corresponding trials. The predicted postures are compared with reconstructed postures focusing on body point coordinates. The posture reconstruction was manually performed on manikins superimposed by images taken in the experiments (Figure 10).

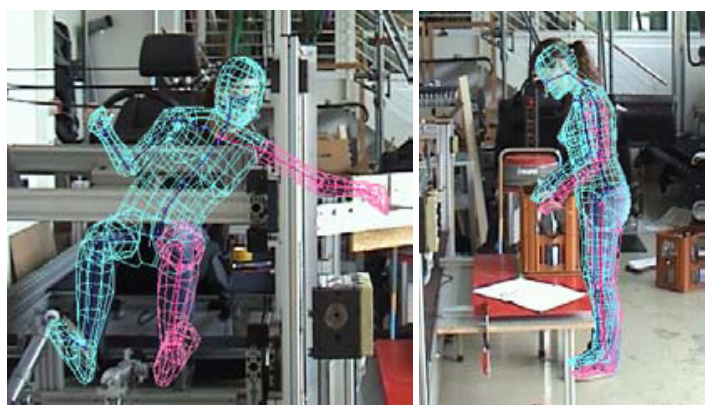

Figure 10 Posture reconstruction by superimposing

The collected body part discomfort ratings showed large discrepancies in the sensibility of the CP50 scale between the subjects. In order to compare these results, the discomfort ratings were normalized with respect to the maximum rating reported for each subject.

\section{Results}

Both model enhancements lead to significant better qualitative prediction results than the original method. The new force coupling restrictions ensure more reliable force configurations. Additionally the enhanced control of the passive receding torques generates more realistic postures for both scenarios.
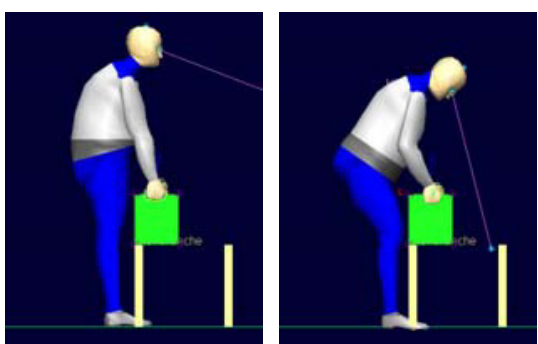

Figure 11 Posture prediction, box (left original, right enhanced)

Figure 12 and Figure 12 show for both scenarios on the left side the posture predicted by the original method and on the right side the enhanced result. The enhanced right postures appear more natural than the original cramped left postures. 

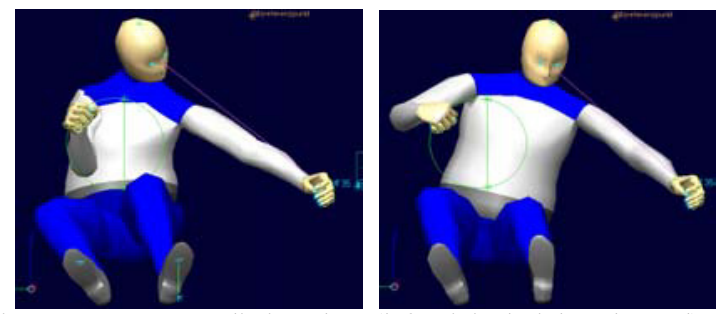

Figure 12 Posture prediction, door (left original, right enhanced)

The numerical comparison of real and predicted body point positions shows different trends for the box and door case.

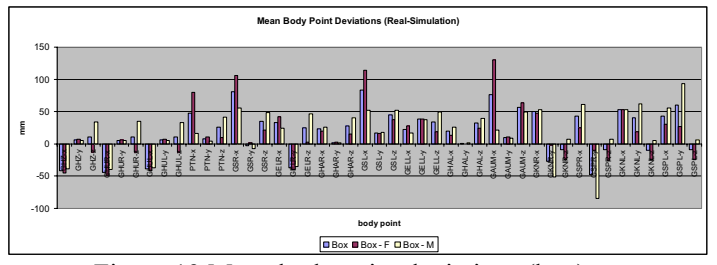

Figure 13 Mean body point deviations (box)

The box case deviations (reality-simulation) are displayed for all relevant body point coordinates gender-independent and -specific (M: males, F: females) in Figure 13. They show that the predicted legs are more splayed and closer to the box than in reality. Furthermore the predicted pelvis is more away from the box and the torso more forwards and downwards. These trends are illustrated for one example in Figure 14. The predicted postures are shown at the left, the real (reconstructed) postures on the right. The green arrows indicate the trends of the prediction with respect to reality.

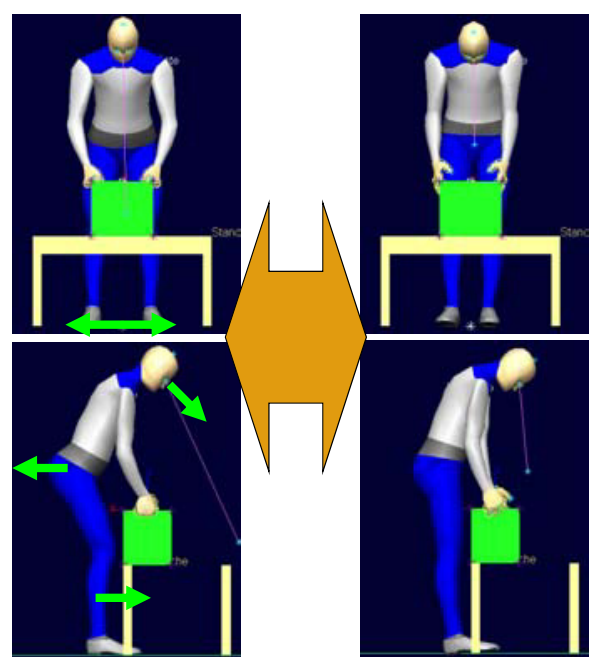

Figure 14 Prediction trends, left prediction, right reality (box)

Analogue to Figure 13 the door case deviations are shown in Figure 15.

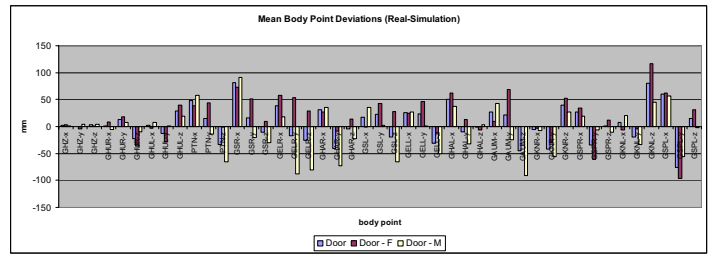

Figure 15 Mean body point deviations (door)

The predicted legs flap more to the right and the feet are more forward and left than in reality. Moreover the predicted head is higher and more right and the torso is bent more forwards. Analogue to Figure 14 these trends are illustrated for one example in Figure 16.
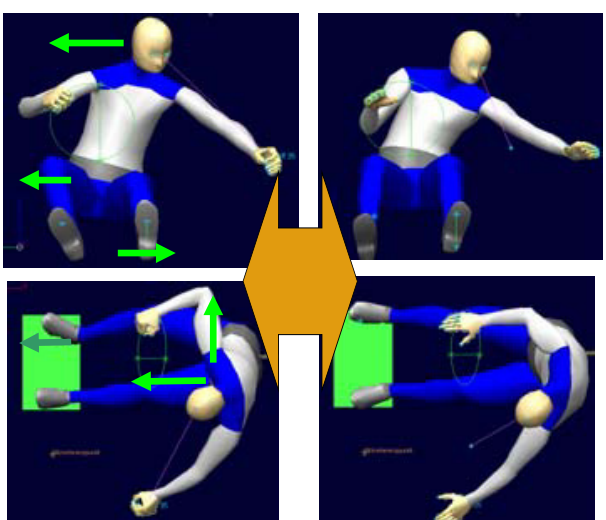

Figure 16 Prediction trends, left prediction, right reality (door)

Finally the validation results of the discomfort prediction are displayed for both scenarios in Figure 17. In particular the mean deviations (realitysimulation) are provided for each relevant body part.

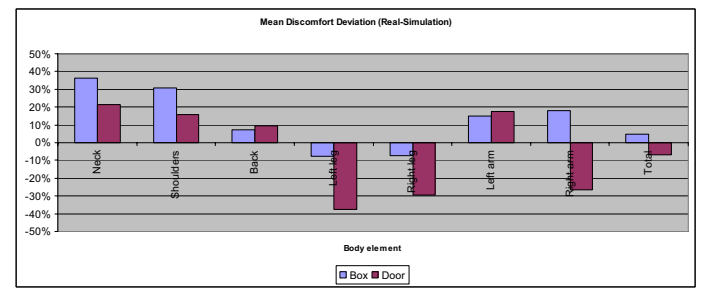

Figure 17 Mean discomfort rating deviations

The diagram show sufficient deviations for the discomfort rating of the back and the total body, but also significant deviations for body parts as neck and legs. 


\section{Discussion}

The presented posture and discomfort prediction approach overcomes the main drawbacks of classical statistical methods:

- Consideration of force and stability aspects That feature enables designers to answer important design questions, which hardly can be handled with available digital tools: Can the occupant adopt a posture to pull the handbrake with a specific force or can the occupant close the door without falling out?

- Compatible posture and discomfort results Since posture and discomfort predictions are based on the same model, the to-be analyzed discomfort criteria are already considered during the posture prediction. This consistency is missing for conventional methods using different prediction models.

- Application to general tasks

Since the approach makes just use of general biomechanical and mechanical models, a wide range of tasks can be simulated and evaluated without additional measurement efforts as required by traditional statistical prediction models.

The prediction results are plausible from a qualitative point of view, but nevertheless, the quantitative validation shows deviations between the prediction and reality. This disadvantage is obvious due to the generality of the method. Task specific prediction models are trained on specific situations and provide smaller deviations, but cannot be applied to other applications.

Hence the presented approach provides a solution for applications, for which no other tools are available and the accuracy plays no critical role.
For precise critical applications the current approach has to be improved. In principle this can be on two levels:

- Experimental joint torque data

Since the required measurements (Figure 3) are quite extensive, the amount of measured subjects could be not representative. The observed deviation trends of the validation suggest that the predicted manikins behave somehow "weaker" than in reality. This could be a proper starting point to investigate and extend the experimental database.

- $\quad$ Prediction models

The experimental data are used for the predictions under several model assumptions. They have to be investigated and checked if they cause the "weaker" behavior prediction mentioned above. This can be supported by the extensive data collected in the validation experiments.

\section{References}

[1] A. Seidl, Das Menschmodell RAMSIS: Analysis, Synthese und Simulation dreidimensionaler Körperhaltungen des Menschen, Dissertation, Technical University Munich, 1994.

[2] A. Marach, CAD-fähiges Prognosemodell für statische Körperhaltungen des Menschen, Dissertation, Technical University Munich, 1999.

[3] J. Kolling, Validierung und Weiterentwicklung eines CADMenschmodells für die Fahrzeuggestaltung, Dissertation, Technical University Munich, 1997.

[4] M. Reed, M. Manary, C. Flannagan and L. Schneider, A Statistical Method for Predicting Automobile Driving Posture, Human Factors 44 No4, Winter 2002.

[5] R. Krist, Modellierung des Sitzkomforts - Eine experimentelle Studie, Dissertation, University of Eichstätt, 1994.

[6] T. Seitz, D. Recluta, D. Zimmermann and H.-J. Wirsching, FOCOPP - An Approach for a Human Posture Prediction Model Using Internal/External Forces and Discomfort, SAE Digital Human Modelling Conference 2005, Paper 2005-012694. 